\title{
BMJ Open CYCLE pilot: a protocol for a pilot randomised study of early cycle ergometry versus routine physiotherapy in mechanically ventilated patients
}

Michelle E Kho, ${ }^{1,2,3}$ Alexander J Molloy, ${ }^{2}$ France Clarke, ${ }^{4}$ Margaret S Herridge, ${ }^{5}$ Karen K Y Koo, ${ }^{6,7}$ Jill Rudkowski, ${ }^{8}$ Andrew J E Seely, ${ }^{9}$ Joseph R Pellizzari, ${ }^{10,11}$ Jean-Eric Tarride, ${ }^{4,12}$ Marina Mourtzakis, ${ }^{13}$ Timothy Karachi, ${ }^{8}$ Deborah J Cook, ${ }^{3,8}$ the Canadian Critical Care Trials Group

To cite: Kho ME, Molloy AJ, Clarke F, et al. CYCLE pilot: a protocol for a pilot randomised study of early cycle ergometry versus routine physiotherapy in mechanically ventilated patients. BMJ Open 2016;6: e011659. doi:10.1136/ bmjopen-2016-011659

- Prepublication history for this paper is available online. To view these files please visit the journal online (http://dx.doi.org/10.1136/ bmjopen-2016-011659).

Received 24 February 2016 Accepted 21 March 2016

CrossMark

For numbered affiliations see end of article.

Correspondence to Michelle E Kho; khome@mcmaster.ca

\section{ABSTRACT}

Introduction: Early exercise with in-bed cycling as part of an intensive care unit (ICU) rehabilitation programme has the potential to improve physical and functional outcomes following critical illness. The objective of this study is to determine the feasibility of enrolling adults in a multicentre pilot randomised clinical trial (RCT) of early in-bed cycling versus routine physiotherapy to inform a larger RCT.

Methods and analysis: 60-patient parallel group pilot RCT in 7 Canadian medical-surgical ICUs. We will include all previously ambulatory adult patients within the first $0-4$ days of mechanical ventilation, without exclusion criteria. After informed consent, patients will be randomised using a web-based, centralised electronic system, to $30 \mathrm{~min}$ of in-bed leg cycling in addition to routine physiotherapy, 5 days per week, for the duration of their ICU stay (28 days maximum) or routine physiotherapy alone. We will measure patients' muscle strength (Medical Research Council Sum Score, quadriceps force) and function (Physical Function in ICU Test (scored), $30 \mathrm{~s}$ sit-tostand, 2 min walk test) at ICU awakening, ICU discharge and hospital discharge. Our 4 feasibility outcomes are: (1) patient accrual of 1-2 patients per month per centre, (2) protocol violation rate $<20 \%$, (3) outcome measure ascertainment $>80 \%$ at the 3 time points and (4) blinded outcomes ascertainment $>80 \%$ at hospital discharge. Hospital outcome assessors are blinded to group assignment, whereas participants, ICU physiotherapists, ICU caregivers, research coordinators and ICU outcome assessors are not blinded to group assignment. We will analyse feasibility outcomes with descriptive statistics.

Ethics and dissemination: Each participating centre will obtain local ethics approval, and results of the study will be published to inform the design and conduct of a future multicentre RCT of in-bed cycling to improve physical outcomes in ICU survivors.

Trial registration number: NCT02377830; Pre-results.

\section{Strengths and limitations of this study}

- In-bed cycling is a novel technology that can improve intensive care unit (ICU) patients' function at hospital discharge if started 2 weeks after ICU admission; however, its effects are unknown when started earlier in a patient's ICU stay to address the rapid muscle weakness due to bed rest.

- The CYCLE pilot is a 60-patient parallel group randomised clinical trial (RCT) of early in-bed cycling versus routine physiotherapy in seven Canadian medical-surgical intensive care units

- We will assess the feasibility of patient accrual, in-bed cycling protocol delivery, outcome measure ascertainment at each of three time points, and blinded outcomes ascertainment at hospital discharge.

- This is a feasibility trial, and is not powered to determine treatment effectiveness.

- Results of the CYCLE pilot will inform the design and conduct of a future multicentre RCT of in-bed cycling to improve physical outcomes in ICU survivors.

\section{INTRODUCTION}

Surviving critical care is the first step in a long road of physical, cognitive and psychological recovery. ${ }^{1}$ While medical advances have reduced the mortality of critical illness, ${ }^{23}$ survival comes with substantial residual physical burdens and societal cost. Intensive care unit (ICU) survivors are at risk of important mobility impairments posthospital discharge. At 1-year follow-up, $34 \%$ of patients surviving their stay in the ICU were below sex-expected and age-expected norms for the 6 min walk test (6MWT), and $51 \%$ had not returned to work. ${ }^{4}$ Although early rehabilitation would benefit survivors if their physical function or 
quality of life could be improved, critically ill mechanically ventilated (MV) patients are often perceived as 'too sick' for physiotherapy (PT) ${ }^{5}$ Rehabilitation in the ICU can be infrequent (eg, $<6 \%$ of all ICU days ${ }^{6}$ ), and when provided, may occur late in a patient's ICU stay (eg, median 10 days post-ICU admission ${ }^{7}$ ).

Patients who develop ICU-acquired weakness are at an increased risk of higher mortality, longer duration of MV, ICU, and hospital length of stay (LOS), and higher hospital costs. ${ }^{8}$ Up to $87 \%$ of MV ICU patients have electrophysiological evidence of neuromuscular abnormalities, ${ }^{9}$ and $55 \%$ have clinically evident weakness. In a study conducted within the first 7 days of ICU admission, patients' quadriceps twitch tension (an involuntary objective measure of muscle force) was four times lower than in healthy controls $(\mathrm{p}<0.001) .{ }^{10}$ After 10 days of ICU admission and MV, quadriceps size decreased almost $18 \%$ from baseline. ${ }^{11}$ Many previously ambulatory patients are unable to walk at ICU discharge due to profound muscle weakness. ${ }^{7}$

A recent systematic review of 14 randomised clinical trials (RCTs) demonstrated that ICU-based exercise studies were most effective to improve long-term physical function in critically ill adults compared with other strategies, including nutrition and different modes of MV. ${ }^{12}$ Early mobility interventions started within the first $48 \mathrm{~h}$ of MV are feasible and can improve function; however, these studies primarily enrolled young medical ICU patients with acute respiratory failure ${ }^{13} 14$ and may not be generalisable to a medical-surgical population or older adults with multiple comorbidities. Cycle ergometry is a promising early ICU exercise intervention for MV patients because it targets the legs, can occur in bed while patients are sedated ${ }^{15}$ or awake, is easily reproducible, and is human resource efficient. In a single-centre, 90-patient RCT, those receiving in-bed leg cycling and usual PT compared with usual PT alone achieved 6MWT distances of 196 vs $143 \mathrm{~m} \quad(\mathrm{p}<0.05)$, had greater leg strength and had better Short Form 36 (SF-36) physical function scores at hospital discharge. ${ }^{16}$ In this study, however, cycling did not start until 2 weeks after ICU admission, ${ }^{16}$ which potentially missed an opportunity to address the early rapid muscle atrophy and deconditioning associated with bed rest in the ICU. ${ }^{11}$

Emerging evidence suggests cycling can occur safely very early in a patient's ICU stay, even while receiving $\mathrm{MV}^{17}{ }^{18}$ A case series of single in-bed cycling sessions started within the first $72 \mathrm{~h}$ of MV showed no increases in cardiac output, oxygen consumption ${ }^{18}$ or safety concerns, even while patients received low-dose vasoactive infusions. ${ }^{18}$ A case-control study enrolling patients within the first $96 \mathrm{~h}$ of MV initiated cycling within $15.3 \mathrm{~h}$ of recruitment. ${ }^{17} 18$ Most recently, a retrospective review of 186 patients and 541 in-bed cycling sessions reported use of in-bed cycling within the first 4 days of ICU admission, with patients receiving a median of two cycling sessions of four total PT sessions. ${ }^{19}$ However, there has been no systematic evaluation of early in-bed leg cycling on functional outcomes in MV patients. The long-term goal of this research programme is to evaluate whether early exercise with in-bed leg cycling, started within 4 days of MV, improves clinically important outcomes. Before embarking on a large-scale trial, a pilot RCT is needed to determine the feasibility of intervention delivery and outcomes assessment in multiple centres. ${ }^{20}$ Here, we report our pilot RCT protocol according to SPIRIT $^{21}$ and TIDieR $^{22}$ guidelines.

\section{OBJECTIVES}

\section{Hypothesis}

It is feasible to enrol adults ( $\geq 18$ years), execute study procedures and measure functional outcomes in a multicentre pilot randomised study of early in-bed cycling versus routine PT to inform a larger RCT. Specifically:

1. Accrual: The overall average accrual rate will be 1-2 patients per month per site.

2. Protocol violations: The in-bed cycling protocol can be successfully implemented with $<20 \%$ protocol violations.

3. Outcome measures: $>80 \%$ of outcomes will be measured as scheduled at three time points: ICU awakening, ICU discharge and hospital discharge.

4. Blinded outcome assessment: $>80 \%$ of physical strength and function outcomes at hospital discharge will be assessed by personnel blinded to group allocation.

\section{METHODS AND ANALYSIS}

\section{Trial design}

The CYCLE pilot RCT is an open-label, concealed study in seven Canadian academic medical-surgical ICUs with blinded outcome assessment at hospital discharge. Table 1 outlines the schedule of enrolment, interventions and assessments.

\section{Participants}

Sixty adults in participating medical-surgical ICUs meeting eligibility criteria will be recruited. All participating ICUs will have a dedicated in-bed cycle ergometer, experience contributing to multicentre critical care trials and a site principal investigator (PI) from the Canadian Critical Care Trials Group. Inclusion criteria: adults ( $\geq 18$ years old) admitted within the first 4 days of MV and first 7 days of ICU, and able to ambulate independently before hospital admission (with or without a gait aid). We chose this timeframe to address the early and rapid muscle atrophy that occurs within the first week of ICU admission. ${ }^{11}$ Exclusion criteria: acute condition impairing patients' ability to cycle (eg, leg fracture), proven or suspected neuromuscular weakness affecting the legs (eg, stroke or Guillain-Barré syndrome), unable to follow commands in English, temporary pacemaker, expected hospital mortality $>90 \%$, body habitus unable to fit the bike, palliative goals of care or persistent therapy exemptions in the first 4 days of MV (see box 1 ). We excluded patients unable to follow commands at 
Table 1 CYCLE pilot RCT schedule of enrolment, interventions and assessments

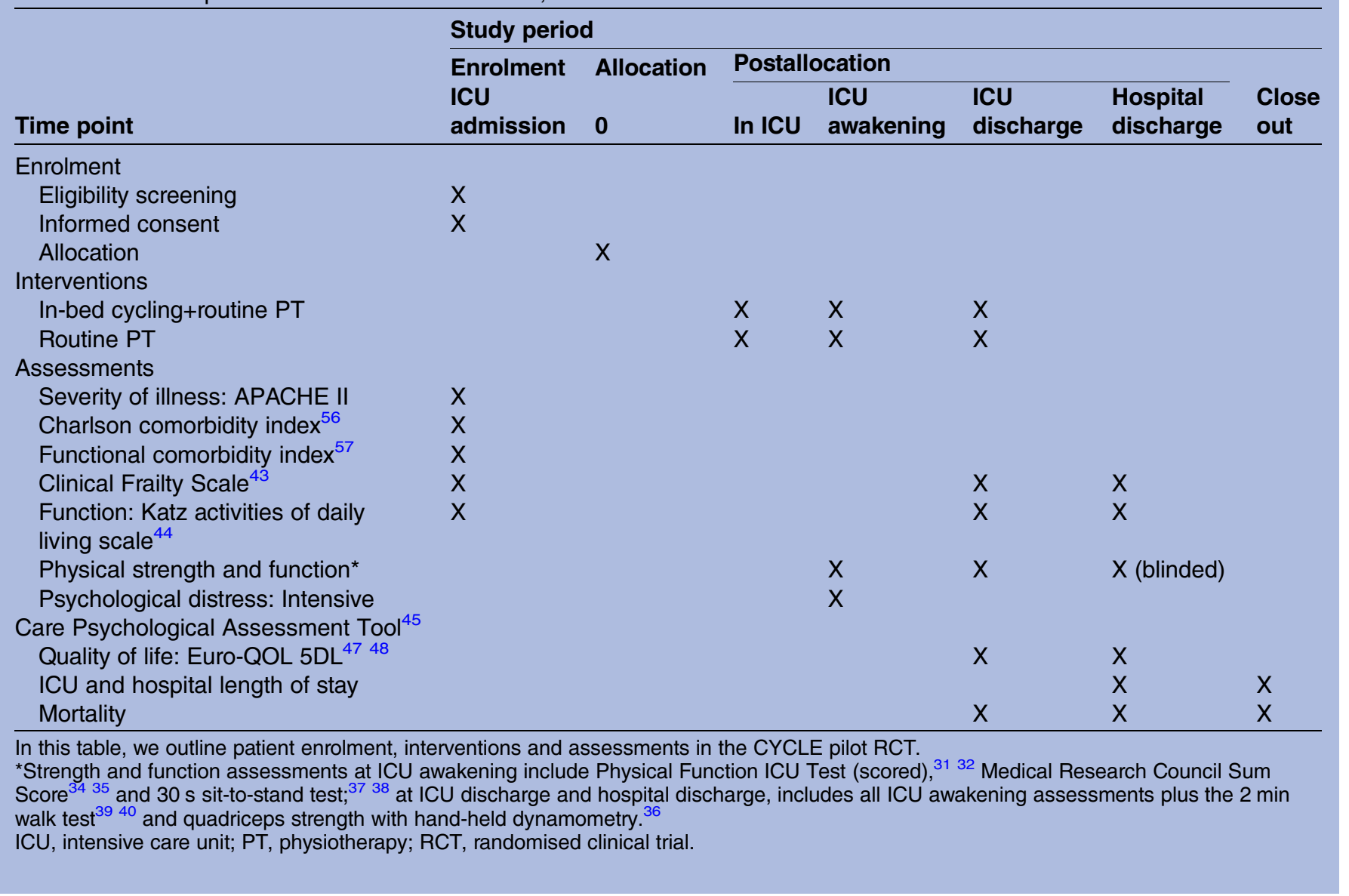

baseline because participants will need to follow simple commands to complete outcome assessments. We excluded patients with conditions associated with muscle weakness to ensure effects of cycling are not confounded by other reasons for persistent muscle weakness.

\section{Recruitment and randomisation}

Enrolment began in March 2015, and is anticipated to continue until December 2016. In each centre, an ICU research coordinator will screen the ICU census regularly to identify patients who meet study criteria and will seek written informed consent from patients or their substitute decision makers before randomisation. Once patients are alert, they will be evaluated for capacity and consented for continuation in the trial. We will use a centralised web-based, secure randomisation service for clinical trials (http://www.randomize.net/). Following consent, the research coordinator will $\log$ in to the website, register the patient and receive the randomised assignment. We will stratify by centre, medical versus surgical admission status and age $\geq 65$ or $<65$ years.

\section{Procedures}

Figure 1 presents the planned flow of participants throughout the study. Individual patients will receive the randomised intervention 5 days per week (excluding weekends and statutory holidays), for the duration of their index ICU stay (maximum 28 days, whichever occurs first) from ICU physiotherapists as part of their normal role. After 28 days, all patients remaining in the ICU will receive routine PT per institutional standards. Those randomised to routine $\mathrm{PT}$ will not receive in-bed cycling. We will conduct outcome assessments at ICU awakening, ICU discharge and hospital discharge (described further below). During PT sessions, physiotherapists will screen participants for readiness for awakening assessments, and will initiate their strength and function assessment once patients successfully answer $\geq 3 / 5$ standardised questions per previous studies (open (close) your eyes; look at me; open your mouth and stick out your tongue; nod your head; raise your eyebrows when I have counted up to 5)..$^{23}$

\section{Experimental}

Patients will receive $30 \mathrm{~min}$ of in-bed cycling in addition to routine PT, for the duration of their index ICU stay (maximum of 28 days or when able to march on the spot for 2 consecutive days with assistance, whichever occurs first). We chose to discontinue cycling after marching on the spot for 2 days to allow physiotherapists and patients to focus on progressing mobility and ambulation activities. During in-bed cycling, patients will be positioned semirecumbently ${ }^{16}$ as per ventilatorassociated pneumonia prevention guidelines. ${ }^{24} 25$ 


\section{Box 1 Daily exemption and in-session stopping criteria}

A. Daily exemption criteria for in-bed cycling

Cycling or physiotherapy (PT) sessions will not occur if any of the following conditions are present:

1. Any increase in vasopressor/inotrope within past $2 \mathrm{~h}$

2. Active myocardial ischaemia, or unstable/uncontrolled arrhythmia per intensive care unit (ICU) team

3. Mean arterial pressure (MAP) $<60$ or $>110 \mathrm{~mm} \mathrm{Hg}$ or out of range for this patient within the past $2 \mathrm{~h}$

4. Heart rate $<40$ or $>140 \mathrm{bpm}$ within the past $2 \mathrm{~h}$

5. Persistent $\mathrm{SpO}_{2}<88 \%$ or out of range for this patient within the past $2 \mathrm{~h}$

6. Neuromuscular blocker within past $4 \mathrm{~h}$

7. Severe agitation (Richmond Agitation and Sedation Scale $>2$ (or equivalent)) within past $2 \mathrm{~h}$

8. Uncontrolled pain

9. Change in goals to palliative care

10. Team perception that in-bed cycling or therapy is not appropriate despite absence of above criteria (eg, active major haemorrhage from any site, acute peritonitis, new incision or wound precluding cycling, new known/suspected muscle inflammation (eg, rhabdomyolysis))

11. Patient or proxy refusal

B. Criteria to terminate in-bed cycling or routine PT Cycling or routine PT will stop if the following occurs:

- Concern for myocardial ischaemia or suspected new unstable/ uncontrolled arrhythmia

- Unplanned extubation

- Physiotherapist perceives continuing cycling or routine PT is not appropriate, for example,

- Respiratory - sustained $\mathrm{O}_{2}$ desaturation $<88 \%$; marked ventilator dysynchrony

- Cardiovascular-sustained symptomatic bradycardia $(<40 \mathrm{bpm})$, tachycardia (>140 bpm), hypotension (MAP $<60 \mathrm{~mm} \mathrm{Hg}$ ) or hypertension (MAP $>120 \mathrm{~mm} \mathrm{Hg}$ )

- Catheter or tube dislodgement or severe patient agitation

- ICU physician, patient or proxy requests termination of session

We will use a specialised in-bed cycle ergometer (eg, RT300 supine cycle), which provides three possible cycling modes: passive (ie, no patient initiation), active-assisted (ie, partially initiated by the patient) or active (ie, fully initiated by the patient). ${ }^{16}$ Our aim is for participants to complete as much active cycling as possible during each $30 \mathrm{~min}$ session. Each patient will receive a pre-programmed standardised treatment template. Each session will start with a 1 min motor-driven passive cycling warm-up at a rate of 5 revolutions per minute (RPM). We chose 5 RPM based on clinical experience with comatose patients who demonstrated some active cycling above the set motor rate. Patients will continue with passive, active-assisted or active cycling for the next $29 \mathrm{~min}$, according to their level of participation. The session will finish with a 30 s motor-driven cool-down (30:30 total). Since ICU patients' level of consciousness may vary throughout their stay, we will allow patients to cycle at a self-selected RPM and will not change the resistance. If the patients stop cycling actively, the ergometer will revert to passive cycling. Therapists will titrate the motor speed to provide sufficient support to promote as much active cycling as possible.

Because of the dynamic nature of critical illness, we will screen participants daily for criteria precluding in-bed cycling (box 1). For example, we will not cycle on a day where a patient has cardiac or respiratory instability, active major bleeding, or severe agitation. During every cycling session, patients will be carefully monitored for safety and indications for termination of cycling, including signs of cardiac or respiratory instability, and catheter or tube dislodgement. We will record vital signs (eg, heart rate), physiological parameters (eg, minute ventilation) and cycling achievements (eg, active cycling, distance) every session. Box 1 outlines cycling session termination criteria.

For centres with no experience with in-bed cycling or with the study bike, we will provide all ICU PTs with a 1-day (approximately $8 \mathrm{~h}$ ) training session on use of the in-bed cycle ergometer from the study PI (MEK) and equipment vendor. This training session includes didactic lectures and use of the cycle with both simulated and critically ill patients. The PTs receive a binder including key ICU rehabilitation trials, specialised ICU bike instruction manuals, a laminated bike quick start pocket card and a computer tablet in a military-grade protective case compliant with hospital infection-control requirements preloaded with electronic versions of all paper materials. Centres will gain clinical experience with routine use of the in-bed cycle with critically ill patients before enrolling patients in the CYCLE pilot RCT. All PTs will receive in-service training on the in-bed biking protocol. At each site, we will train multiple PTs to bike to ensure a trained therapist is always available despite vacation time or unplanned absences. The Methods Centre will also assist each site with trouble-shooting equipment problems and outcome measure questions.

\section{Control: routine PT}

Patients will receive routine PT per current institutional practice as part of their normal role. Routine PT may include activities to assist with optimising airway clearance and respiratory function, and, based on the patient's alertness and medical stability, activities to maintain or increase limb range of motion and strength, in and out of bed mobility, and ambulation. ${ }^{13} 142627$ We expect some interinstitutional variation in routine PT interventions. To date, there are no Canadian data documenting routine PT interventions; two point prevalence studies $^{28} 29$ and a multicentre prospective cohort study documented inconsistent mobilisation practices in different countries, across centres. ${ }^{30}$ We will use the same criteria to terminate routine PT sessions (box 1).

\section{Outcome measures}

The four feasibility outcomes are outlined above. Below, we describe the planned primary and secondary 
Figure 1 Planned flow of participants throughout the CYCLE pilot RCT. ADL, activities of daily living; ICU, intensive care unit; MV, mechanically ventilated; PT, physiotherapy; RCT, randomised clinical trial.

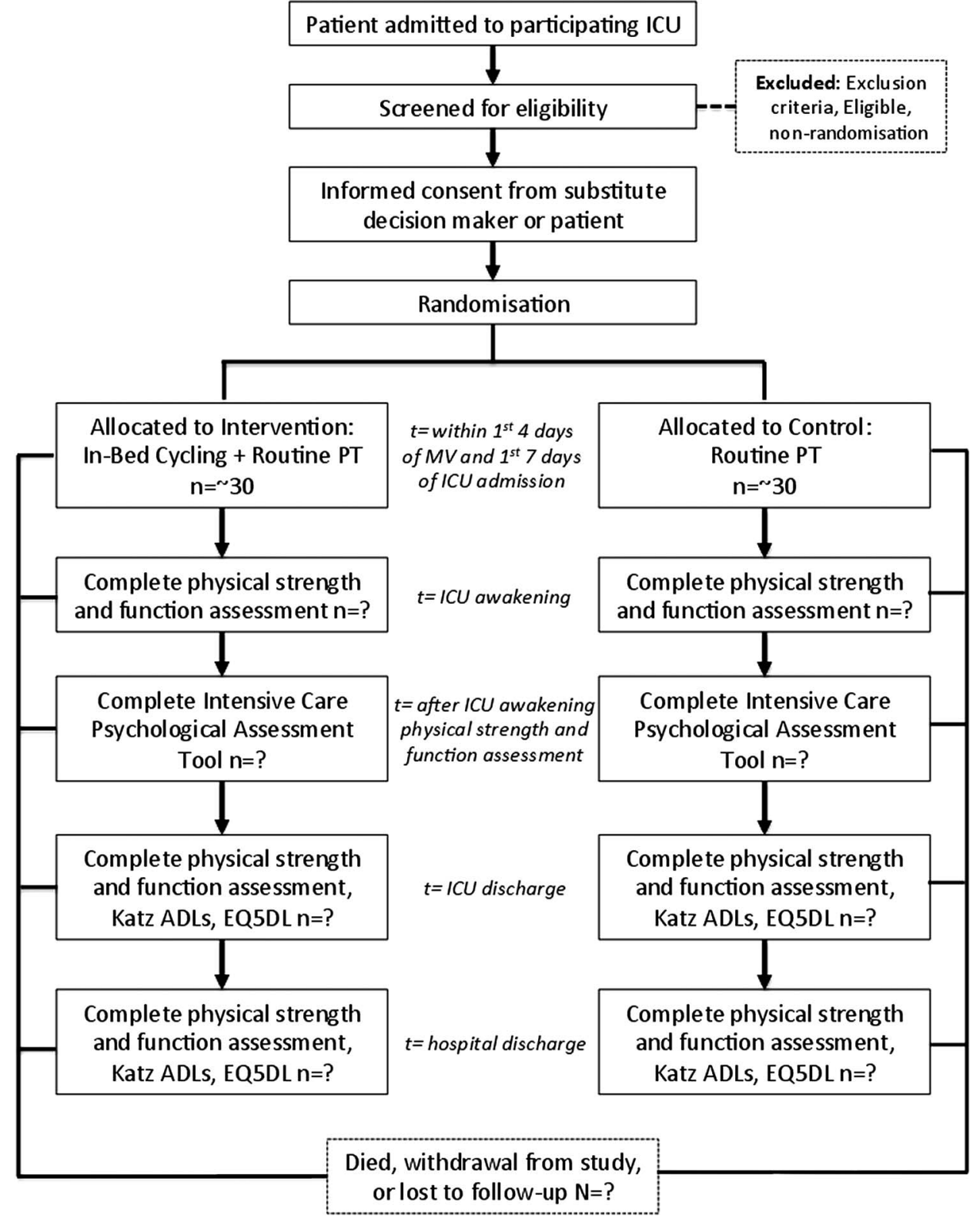

outcome measures for the full CYCLE RCT. We will measure all of the outcomes described below in the CYCLE pilot RCT.

Outcome measures for the full CYCLE RCT: The primary outcome for the full RCT will be the Physical Function ICU Test-scored (PFIT-s) measured at hospital discharge. ${ }^{31}{ }^{32}$ It is a reliable and valid four-item scale (arm and leg strength, ability to stand, and step cadence) with a score range from 0 to 10 (higher scores=better function). ${ }^{31}{ }^{32}$ We chose the PFIT-s because we expect all ICU patients will be able to complete part of the assessment even if they cannot stand (eg, arm or leg strength), limiting floor effects, ${ }^{33}$ and its strong psychometric properties (reliability intraclass correlation coefficient range $=0.996-1.00^{32}$; convergent validity with the $6 \mathrm{MWT}$ and manual muscle strength testing ${ }^{31}$ ).

Secondary outcomes in the full CYCLE RCT include muscle strength (Medical Research Council manual muscle strength, ${ }^{34}{ }^{35}$ quadriceps strength ${ }^{36}$ ) and function (eg, 30 s sit-to-stand, ${ }^{37} 38$ and 2 min walk test). ${ }^{39} 40$ These measures have normative values, good reliability in critically ill or frail elderly populations and are included in other ongoing ICU rehabilitation studies. ${ }^{41}{ }^{42}$ We will also collect hospital discharge location, frailty, ${ }^{43}$ length of MV, LOS (ICU, hospital) and mortality (ICU, hospital), patients' perception of physical function, Katz activities of daily living (ADLs) scale, ${ }^{44}$ critical care-related psychological distress (Intensive Care Psychological Assessment Tool (IPAT) ${ }^{45}$ ), and health-related quality of life (EQ-5DL). ${ }^{47-49}$ table 2 describes the outcome measures.

We will follow all patients throughout their ICU and hospital stay until death, transfer to another hospital or hospital discharge. At each site, a research coordinator will track each patient's location in hospital and liaise with hospital staff to identify anticipated hospital discharge date. At ICU discharge and at hospital discharge, the research coordinator will assess patients' perceptions 
Table 2 Description of outcome measures for the CYCLE pilot and full RCT

\begin{tabular}{|c|c|}
\hline Outcome measure & Description \\
\hline \multicolumn{2}{|c|}{ Physical strength and function measures } \\
\hline $\begin{array}{l}\text { 1. Outcome for full RCT } \\
\text { (anticipated): PFIT-s }{ }^{31} 32\end{array}$ & $\begin{array}{l}\text { Patients complete four activities: arm and leg strength, ability to stand, and step } \\
\text { cadence. Scores range from } 0 \text { to } 10 \text {, with higher score=better function. }\end{array}$ \\
\hline \multicolumn{2}{|l|}{ 2. Outcomes } \\
\hline $\begin{array}{l}\text { Medical Research Council } \\
\text { muscle strength }\end{array}$ & $\begin{array}{l}\text { Standardised physical examination of six muscle groups (three upper, three lower), } \\
\text { using a six-point scale }(0=\text { no contraction; } 5=\text { contraction sustained against maximal } \\
\text { resistance), summed to a total score (range } 0-60) \text {, higher score=better strength. }\end{array}$ \\
\hline 30 s sit-to-stand ${ }^{37} 38$ & $\begin{array}{l}\text { Patient completes as many full sit-to-stand repetitions within } 30 \mathrm{~s} \text {, with higher } \\
\text { score=better strength. }\end{array}$ \\
\hline 2 min walk test 3940 & Patient walks as far as possible over 2 min, with farther distance=better endurance. \\
\hline Quadriceps strength ${ }^{36}$ & $\begin{array}{l}\text { Standardised physical examination using a small device that fits into the palm of the } \\
\text { examiner's hand, and quantifies force (in Newtons) on a continuous scale when the } \\
\text { patient's leg pushes against the device, higher score=better strength. }\end{array}$ \\
\hline \multicolumn{2}{|l|}{ Other measures } \\
\hline Clinical Frailty Scale ${ }^{43}$ & $\begin{array}{l}\text { Nine-point scale evaluating physical function, activities of daily living, instrumental } \\
\text { activities of daily living and assistance for personal care; higher score=more frailty and } \\
\text { poorer function. }\end{array}$ \\
\hline $\begin{array}{l}\text { Katz activities of daily living } \\
\text { scale }^{44}\end{array}$ & $\begin{array}{l}\text { Six-question survey evaluating dependence or independence in bathing, dressing, } \\
\text { toileting, transferring, continence and feeding. Each item rated dependent or } \\
\text { independent; higher score=more independence. }\end{array}$ \\
\hline $\begin{array}{l}\text { Intensive Care Psychological } \\
\text { Assessment Tool }^{45}\end{array}$ & $\begin{array}{l}\text { Ten-item interviewer-administered questionnaire to identify acute distress and risk of } \\
\text { future psychological distress. Score ranges from } 0 \text { to } 20 \text {; score of } 7 \text { or more represents } \\
\text { higher risk of psychological distress. }\end{array}$ \\
\hline Quality of Life: EuroQOL 5DL ${ }^{47} 48$ & $\begin{array}{l}\text { Five-question interviewer or self-administered, preference-based instrument to } \\
\text { measure mobility, self-care, usual activities, pain and anxiety/depression, and a global } \\
\text { assessment of health; higher score=better quality of life. }\end{array}$ \\
\hline
\end{tabular}

of physical function, Katz ADL scale, ${ }^{44}$ IPAT $^{45}{ }^{46}$ and EQ-5DL. 4748

All strength and physical function outcome assessors will receive a $3 \mathrm{~h}$ in-person training session and support materials. At each site, we will train multiple assessors to ensure a blinded outcomes assessor is always available despite planned or unplanned absences. This interactive training session includes didactic lectures, and use of the strength and physical function outcome measures with simulated patients. The PTs will receive paper copies of each outcome measure, administration instructions and normative values (where available).

\section{Harms}

We expect few risks to the safety of participants involved in either arm of the CYCLE Pilot RCT. Routine PT in the ICU, including in-bed cycling, is safe. A comprehensive review of 2.5 years of PT in a critical care rehabilitation programme in 1110 patients and over 5267 rehabilitation sessions identified physiological abnormalities or potential adverse events in 2.5 per 1000 patients and 6 per 1000 therapy sessions, respectively. ${ }^{50}$ Of these, patients received 628 in-bed cycling sessions as part of routine PT, and experienced 1 safety event (1.6 safety events per 1000 PT treatment days). In a focused retrospective review of a subset of the critical care rehabilitation programme described above, of 541 cycling sessions, patients experienced one radial arterial catheter dislodgement, no unplanned extubations and no predefined cardiorespiratory physiological abnormalities. ${ }^{51}$ Authors reported no catheter or tube dislodgements in six ICU cycling studies. ${ }^{16-18}{ }^{52-54}$ Similarly, in the RCT of cycling started 2 weeks into the patient's ICU stay, no severe physiological adverse events occurred (eg, arrhythmias, myocardial ischaemia); 16 sessions $(4 \%)$ stopped early due to low oxygen saturation $(<90 \% ; \mathrm{n}=8)$ or blood pressure concerns $(\mathrm{n}=8$, systolic >180 $\mathrm{mm} \mathrm{Hg} ; \mathrm{n}=6,>20 \%$ decrease in diastolic; $\mathrm{n}=2$ ); all variables returned to baseline within $2 \mathrm{~min}$ of activity cessation. ${ }^{16}$ Three patients in the cycling group withdrew: two due to cardiac instability, and one due to an Achilles tendon rupture. ${ }^{16}$ Box 1 outlines termination criteria and safety events recorded in the CYCLE pilot RCT. We will also record the consequences of the safety events.

\section{Blinding}

Given the nature of the intervention, patients, ICU PTs, ICU caregivers, family members and research coordinators will not be blinded to intervention allocation. However, outcomes assessors will be blinded to the allocation, as they will be assessed by a core group of PTs who did not care for patients in the ICU. We will ask patients and their family members not to disclose the patient's assigned treatment to PTs involved in assessing hospital outcomes to protect against performance bias. 


\section{DATA COLLECTION, MANAGEMENT AND ANALYSIS PLAN}

In both groups, we will collect baseline data including patient demographics, ICU admission reason, medical versus surgical status, severity of illness, ${ }^{55}$ comorbidity ${ }^{56} 57$ and prehospital function. ${ }^{44}$ ICU-related variables captured daily during the patient's ICU stay will include illness severity, ${ }^{55}$ other life supports, drug exposure and nutrition. We will collect relevant cointerventions that may impair patient function, including receipt of corticosteroids $^{58}$ and neuromuscular blocking agents, ${ }^{59}$ and duration of bed rest. ${ }^{59}$ We will also record the type and duration of all PT interventions (eg, passive or active range of motion, bed mobility and transfers, ambulation) received in the ICU.

\section{STATISTICS}

\section{Sample size calculation}

We will recruit 60 patients for this pilot RCT. Our sample size calculation is based on identifying a 0.25 standardised effect size for the full RCT for the PFIT-s at hospital discharge. ${ }^{31}$ Assuming a baseline SD of 3.06 points at ICU awakening, ${ }^{31}$ we hypothesise that 0.75 points in the final PFIT-s score at hospital discharge is clinically important for the main trial. Using a CI approach for continuous outcomes, we require 504 participants with outcomes at hospital discharge to detect a difference in the main trial $(\alpha=0.05) .{ }^{60}$ For the pilot RCT, we will recruit $9 \%$ of the sample size for the planned main trial to have an $80 \%$ power to detect such a difference. ${ }^{60}$ Thus, we need to recruit, randomise and analyse 46 patients (23 per group) to produce a one-sided $80 \%$ confidence limit, which would exclude a 0.75 difference on the PFIT if the point estimate from the pilot study were 0 . Assuming $25 \%$ in-hospital mortality, we plan to include 60 patients in the pilot RCT.

\section{STATISTICAL ANALYSIS}

For all feasibility analyses, we will include all patients randomised, regardless of protocol adherence. We will conduct a subgroup analysis of patients $\geq 65$ years old. Since elderly patients are under-represented in critical care trials, ${ }^{61}$ and no studies have specifically studied early cycling in the elderly critically ill, ${ }^{62}$ subgroup analysis of these patients for our four primary objectives will help to identify barriers and facilitators to conducting the research protocol in this population. We will have no formal interim analysis in this pilot trial. We will use data from the CYCLE pilot RCT in the full CYCLE RCT and will consider public access to data and statistical code after the full RCT. Table 3 outlines the variables, hypotheses, outcome measures and analytic methods for our four feasibility outcome measures.

\section{Trial management}

The Methods Centre, coordinated by St Joseph's Healthcare and McMaster University, will oversee all contracts, research ethics board preparation, site initiation and training, screening $\log$ and data submission, data quality assurance, study close-out, and finances at each site. It will develop and prepare all study materials (eg, standard operation procedures, operations manuals, data collection forms) for participating sites, be the

Table 3 CYCLE pilot RCT variables, measures and methods of analysis for the four feasibility objectives

\begin{tabular}{|c|c|c|c|}
\hline Variable/outcome & Hypothesis & Outcome measure & $\begin{array}{l}\text { Methods of } \\
\text { analysis }\end{array}$ \\
\hline \multicolumn{4}{|l|}{ Feasibility outcomes } \\
\hline 1. Accrual & $\begin{array}{l}\text { The overall average accrual rate will } \\
\text { be } 1-2 \text { patients per month per site. }\end{array}$ & $\begin{array}{l}\text { Average monthly patient enrolment per } \\
\text { site }\end{array}$ & $\begin{array}{l}\text { Descriptive statistics } \\
\text { (mean, SD) by site }\end{array}$ \\
\hline $\begin{array}{l}\text { 2. Protocol } \\
\text { violations }\end{array}$ & $\begin{array}{l}\text { The in-bed cycling protocol can be } \\
\text { successfully implemented with }<20 \% \\
\text { protocol violations. }\end{array}$ & $\begin{array}{l}\text { (1) Patients with no cycling exemptions } \\
\text { from box } 1 \text { who did not receive cycling, } \\
\text { and(2) Patients with cycling } \\
\text { exemptions from box } 1 \text { and did receive } \\
\text { cycling }\end{array}$ & $\begin{array}{l}\text { Descriptive statistics } \\
(\mathrm{n}, \%, 95 \% \mathrm{Cl})\end{array}$ \\
\hline $\begin{array}{l}\text { 3. Outcome } \\
\text { measures }\end{array}$ & $\begin{array}{l}>80 \% \text { of outcomes (described above) } \\
\text { will be measured as scheduled at ICU } \\
\text { awakening, ICU discharge, and } \\
\text { hospital discharge. }\end{array}$ & $\begin{array}{l}\text { Whether the measurement occurred, } \\
\text { the result, and any barriers to data } \\
\text { collection }\end{array}$ & $\begin{array}{l}\text { Descriptive statistics } \\
(\mathrm{n}, \%, 95 \% \mathrm{Cl})\end{array}$ \\
\hline $\begin{array}{l}\text { 4. Blinded } \\
\text { outcome } \\
\text { assessments }\end{array}$ & $\begin{array}{l}>80 \% \text { of physical strength and } \\
\text { function outcomes at hospital } \\
\text { discharge will be assessed by } \\
\text { personnel blinded to group allocation. }\end{array}$ & $\begin{array}{l}\text { Whether the measurement occurred, } \\
\text { the result, and any barriers to data } \\
\text { collection }\end{array}$ & $\begin{array}{l}\text { Descriptive statistics } \\
(\mathrm{n}, \%, 95 \% \mathrm{Cl})\end{array}$ \\
\hline \multicolumn{4}{|l|}{ Subgroup analysis } \\
\hline $\begin{array}{l}\geq 65 \text { years old and } \\
<65 \text { years old }\end{array}$ & $\begin{array}{l}\text { There is no difference in any of the } \\
\text { above four feasibility objectives } \\
\text { between those } \geq 65 \text { years old and } \\
\text { those }<65 \text { years old }\end{array}$ & As outlined above & $\chi^{2}$ test \\
\hline
\end{tabular}


point contact for study questions, and will communicate important protocol amendments electronically to relevant parties. To protect confidentiality, all data will be anonymised and entered into iDataFax, a passwordprotected encrypted server that runs on Red Hat Enterprise Linux. All PIs will have access to the clean data set and their local data after the full CYCLE RCT.

\section{Steering committee}

The CYCLE pilot RCT steering committee will be a subgroup of co-investigators, including MEK, DJC, all site leads, and the Methods Centre research coordinator. This group will provide input on any necessary protocol revisions, and offer clinical guidance. We will have a formal Data Monitoring Committee for the full CYCLE RCT.

\section{ETHICS AND DISSEMINATION}

We will disseminate study results regardless of the magnitude or direction of effect. We will disseminate results to key stakeholders (eg, critical care clinicians, critical care triallists, research funders and the public) through conference presentations, peer-review journal publications, trial registry (clinicaltrials.gov) and the CYCLE trial website (http://www.icucycle.ca). We will submit trial progress summaries to our sponsors as required. We will not use professional writers and will follow the International Committee of Medical Journal Editors for authorship. ${ }^{63}$

\section{DISCUSSION}

\section{Limitations and strengths of the CYCLE multicentre pilot RCT}

The CYCLE pilot RCT is designed as a feasibility study, and is therefore not powered to determine treatment effectiveness. In-bed cycle ergometry only targets the lower extremities, whereas the upper extremities and torso also weaken with bed rest. ${ }^{64}$ Implementing this cycling protocol as part of their normal role will add to the workload of participating physiotherapists; however, we expect that efficiency with the cycling protocol will improve over time in participating centres, as recorded in our pilot study. Cycling is not necessarily a functionally oriented therapy; however, once patients can march on the spot, therapists will transition from cycling to help patients focus on advancing other mobility activities. Moreover, cycling allows both passive and active activity, which is easily adaptable to a patient's current physical status. Our pilot trial is modest in size but is a foundational step in this research programme.

This study will engage the largest number of ICUs to date in the field of critical care rehabilitation. Numerous strengths of this proposed research include the innovative, portable and publically familiar intervention of cycling. In-bed cycling can occur while patients are deeply sedated, unconscious or are minimally interactive. ${ }^{15}$ Our intervention targets the leg muscles, which account for $75 \%$ of total skeletal muscle mass, ${ }^{65}$ and are most vulnerable to loss of muscle size and strength during bed rest. ${ }^{64}{ }^{66}$ Unlike ambulation during MV, which can require up to four clinicians, ${ }^{67}$ cycling only requires the assistance of one clinician. ${ }^{16}$ Our pilot data support the safety and feasibility of early cycling in critically ill patients receiving $\mathrm{MV}^{68}$ and we are engaging front-line PTs to provide the cycling intervention as part of normal care, in anticipation of future knowledge translation efforts. To reduce detection bias, we will conduct blinded functional outcome assessments at hospital discharge. We will collect key feasibility data to inform a future larger RCT.

Results from the CYCLE pilot RCT will inform the future large-scale multicentre CYCLE RCT. Consistent with the four primary objectives of our pilot RCT, we will identify barriers and facilitators to accrual, including occasions to revise inclusion and exclusion criteria, and improve the informed consent process, if needed. We will systematically collect protocol violations to identify opportunities to optimise and streamline the delivery of in-bed cycling in other centres by seeking direct feedback from the front-line physiotherapists at each site. We will assess our ability to conduct outcome measures at ICU awakening, ICU discharge and hospital discharge, and blinded outcome measures at hospital discharge. Finally, results from our pilot RCT will document the nature and frequency of routine PT interventions in multiple centres in the new era of early mobility activities in critically ill patients.

We anticipate the primary outcome for the full CYCLE RCT will be the PFIT-s, ${ }^{31}$ powered to detect a difference in patients' function at hospital discharge. Thus, our ability to successfully measure outcomes with minimal losses to follow-up is critical. We will collate and synthesise strategies from outcome assessors to maximise our outcome measures. Observed hospital mortality and loss to follow-up data will inform the number of patients we will need to recruit for the full CYCLE RCT to achieve our target sample size at hospital discharge.

By 2026, the number of patients aged $>60$ years requiring MV is expected to increase by $105 \% .^{69}$ This presents an urgent need to proactively address ICU rehabilitation needs, since more of these survivors will be at risk for post-ICU disability. If effective, early in-bed leg cycling could decrease disability and may represent a costeffective healthcare intervention.

\section{Author affiliations}

${ }^{1}$ McMaster University, School of Rehabilitation Science, Hamilton, Ontario, Canada

${ }^{2}$ Department of Physiotherapy, St. Joseph's Healthcare, Hamilton, Ontario, Canada

${ }^{3}$ Department of Physical Medicine and Rehabilitation, Johns Hopkins University, Baltimore, Maryland, USA

${ }^{4}$ Department of Clinical Epidemiology and Biostatistics, McMaster University, Hamilton, Ontario, Canada

${ }^{5}$ Department of Medicine, University of Toronto, Toronto General Research Institute, University Health Network, Toronto, Ontario, Canada

${ }^{6}$ Swedish Early Mobility Program in Critical Care, Swedish Medical Group, First Hill Campus, Seattle, Washington, USA 
${ }^{7}$ Department of Medicine, Western University, London, Ontario, Canada ${ }^{8}$ Department of Medicine, McMaster University, Hamilton, Ontario, Canada ${ }^{9}$ Ottawa Hospital Research Institute, University of Ottawa, Ottawa, Ontario, Canada

${ }^{10}$ Consultation-Liaison Psychiatry Service, St. Joseph's Healthcare, Hamilton, Ontario, Canada

${ }^{11}$ Department of Psychiatry and Behavioural Neurosciences, McMaster University, Ontario, Canada

${ }^{12}$ Programs for the Assessment of Technology in Health, Research Institute of St. Joe's Hamilton, Hamilton, Ontario, Canada

${ }^{13}$ Department of Kinesiology, University of Waterloo, Waterloo, Ontario, Canada

Twitter Follow Michelle Kho at @khome

Acknowledgements The authors are grateful to the following participating sites, site principal investigators (PI), additional co-investigators, physiotherapists (ICU PTs, blinded assessors), research coordinators (RC), research assistants (RA), and students who have or will contribute to the CYCLE pilot RCT: St Joseph's Healthcare: Site PI: MEK; Intensivists: DJC, JR; ICU PTs: Daana Ajami, Laura Camposilvan (PT assistant), MEK, Magda McCaughan, Christina Murphy, Kristy Obrovac, Wendy Perry; Blinded assessors: Diana Hatzoglou, Miranda Prince (PT assistant), Bashir Versi; RCs: AJM, France Clarke, F Aileen Costigan, Marnie Jakab; RAs: Michael Ciancone, Devin McCaskell; PT student: Janelle Unger; Graduate student: Julie Reid; Clinical psychologist: JRP; Co-investigator: Thomas Piraino; Juravinksi Hospital: Site PIs: TK, Bram Rochwerg; ICU PTs: Tania Brittain, Shivaun Davidson, Christopher Farley, Andrea Galli, Leigh Ann Niven, Jessica Temesy; Blinded assessors: Helen Bishop, Shannon Earl (PT assistant), Chelsea Hale, Gillian Manson; RC: Tina Millen; Hamilton General Hospital: Site PI: Alison Fox-Robichaud; ICU PTs: Ashley Eves, Elise Loreto, Anastasia Newman, Judi Rajczak, Julie Reid, Sarah Lohanyai; Blinded assessors: Jennifer Duley, Sue Mahler, Matthew McCaffrey, Jessica Pilon-Bignell; RC: Ellen McDonald; RA: Julie Reid; Toronto General Hospital: Site PIs: Vincent Lo, Sunita Mathur; Intensivist: MSH; ICU PTs: Anne-Marie Bourgeois, Nathalie Côté, Adriane Lachmaniuk, Vincent Lo, Sophie Mendo; Blinded assessors: Gary Beauchamp, Sherry Harburn, Megan Hudson, Teresa Torres; RC: Andrea Matte; RAs: Jaimie Archer, Daniel Chen, Luciana Urache; London Health Sciences: Site PI: Ian Ball; ICU PTs: Kristen Abercombie, Erin Blackwell-Knowles, Jennifer Curry, Tania Larsen; Blinded assessor: Jennifer Jackson; RC: Eileen Campbell; RA: Rebecca Rondinelli St Michael's Hospital: Site PIs: Karen Burns, Jan Friedrich; ICU PTs: Sarah Brown, Deanna Feltracco, Christine Leger; Blinded assessors: Diana Horobetz, Anna Michalski, Verity Tulloch, Natalia Zapata; RCs: Orla Smith, Kurtis Salway, Gyan Sandhu Ottawa General Hospital: Site PI: AJES; ICU PTs: Rachel Goard, Josée Lamontagne; Blinded assessors: Michelle Cummings, Sarah Patten; RCs: Irene Watpool, Rebecca Porteous, Brigette Gomes; RAs: Shelley Acres, Heather Langlois

Collaborators Canadian Critical Cae Trials Group.

Contributors MEK was involved in study conception and manuscript draft, and MEK, FC, MSH, KKYK, JR, AJES, JRP, J-ET and DJC contributed to design. MEK, DJC, KKYK and MSH provided expertise in clinical trial design. MEK, DJC, JR, JRP, J-ET, TK, MSH, AJES and MM are grant holders. This study is part of a research programme supported by the Canadian Critical Care Trials group. All authors contributed to refinement of the study protocol and approved the final manuscript.

Funding This work is supported by grants from Technology Evaluation in the Elderly Network Catalyst (CAT2014-05), Canadian Respiratory Research Network Emerging Research Leaders Initiative, Ontario Thoracic Society Grant-in-Aid, and Canadian Institutes of Health Research Transitional Operating Grant (Award \#142327), Canada Foundation for Innovation, Ontario Ministry of Research and Innovation. MEK and DJC are each funded by a Canada Research Chair. Restorative Therapies (Baltimore, Maryland, USA) provided two RT-300 supine cycle ergometers for Toronto General Hospital and London Health Sciences sites for this research.

Competing interests MEK received an equipment loan of two RT300 supine cycles from Restorative Therapies, Baltimore, Maryland, USA, for this study.
MSH and lan Ball are each site investigator at centres where MEK received the equipment loan from Restorative Therapies.

Disclaimer The funding sources and equipment manufacturer had no role in the design of this study and will not have any role during its execution, analyses, interpretation of the data, or decision to submit results.

Ethics approval The following Research Ethics Boards (REBs) approved this study: Hamilton Integrated REB for St Joseph's Healthcare, Juravinski ICU, and Hamilton General ICU (14-531); Toronto General Hospital (15-9282); London Health Sciences (107202); St. Michael's Hospital (15-334); Ottawa General (20150732-01H).

Provenance and peer review Not commissioned; peer reviewed for ethical and funding approval prior to submission.

Open Access This is an Open Access article distributed in accordance with the Creative Commons Attribution Non Commercial (CC BY-NC 4.0) license, which permits others to distribute, remix, adapt, build upon this work noncommercially, and license their derivative works on different terms, provided the original work is properly cited and the use is non-commercial. See: http:// creativecommons.org/licenses/by-nc/4.0/

\section{REFERENCES}

1. Needham DM, Davidson J, Cohen $\mathrm{H}$, et al. Improving long-term outcomes after discharge from intensive care unit: report from a stakeholders' conference. Crit Care Med 2012;40:502-9.

2. Esteban A, Frutos-Vivar F, Muriel A, et al. Evolution of mortality over time in patients receiving mechanical ventilation. Am J Respir Crit Care Med 2013;188:220-30.

3. Kaukonen KM, Bailey M, Suzuki S, et al. Mortality related to severe sepsis and septic shock among critically ill patients in Australia and New Zealand, 2000-2012. JAMA 2014;311:1308-16.

4. Herridge MS, Tansey CM, Matte A, et al. Functional disability 5 years after acute respiratory distress syndrome. $N$ Engl $J$ Med 2011;364:1293-304.

5. Gosselink R, Bott J, Johnson M, et al. Physiotherapy for adult patients with critical illness: recommendations of the European Respiratory Society and European Society of Intensive Care Medicine Task Force on Physiotherapy for Critically III Patients. Intensive Care Med 2008;34:1188-99.

6. Needham DM, Wang W, Desai SV, et al. Intensive care unit exposures for long-term outcomes research: development and description of exposures for 150 patients with acute lung injury. $J$ Crit Care 2007;22:275-84.

7. Zanni JM, Korupolu R, Fan E, et al. Rehabilitation therapy and outcomes in acute respiratory failure: an observational pilot project. $J$ Crit Care 2010;25:254-62.

8. Hermans G, Van Mechelen H, Clerckx B, et al. Acute outcomes and 1 -year mortality of intensive care unit-acquired weakness. A cohort study and propensity-matched analysis. Am J Respir Crit Care Med 2014;190:410-20.

9. Stevens RD, Dowdy DW, Michaels RK, et al. Neuromuscular dysfunction acquired in critical illness: a systematic review. Intensive Care Med 2007;33:1876-91.

10. Vivodtzev I, Devost A, Saey D, et al. Severe and early quadriceps weakness in mechanically ventilated patients. Crit Care 2014; 18:431.

11. Puthucheary ZA, Rawal J, McPhail M, et al. Acute skeletal muscle wasting in critical illness. JAMA 2013;310:1591-600.

12. Calvo-Ayala E, Khan BA, Farber MO, et al. Interventions to improve the physical function of ICU survivors: a systematic review. Chest 2013;144:1469-80.

13. Morris PE, Goad A, Thompson C, et al. Early intensive care unit mobility therapy in the treatment of acute respiratory failure. Crit Care Med 2008;36:2238-43.

14. Schweickert WD, Pohlman MC, Pohlman AS, et al. Early physical and occupational therapy in mechanically ventilated, critically ill patients: a randomised controlled trial. Lancet 2009;373:1874-82.

15. Kho ME, Molloy AJ, Clarke F, et al. Quantifying active in-bed cycling in medical-surgical critically ill patients. Am J Respir Crit Care Med 2014;189:A3880.

16. Burtin C, Clerckx B, Robbeets C, et al. Early exercise in critically ill patients enhances short-term functional recovery. Crit Care Med 2009;37:2499-505.

17. Parry SM, Berney S, Warrillow S, et al. Functional electrical stimulation with cycling in the critically ill: a pilot case-matched control study. J Crit Care 2014;29:695.e1-e7. 
18. Camargo Pires-Neto R, Fogaca Kawaguchi YM, Sayuri Hirota A, et al. Very early passive cycling exercise in mechanically ventilated critically ill patients: physiological and safety aspects-a case series. PLOS ONE 2013;8:e74182.

19. Kho ME, Martin RA, Toonstra AL, et al. Feasibility and safety of in-bed cycling for physical rehabilitation in the intensive care unit (ICU). J Crit Care 2015;30:e1-5.

20. Arnold DM, Burns KE, Adhikari NK, et al. The design and interpretation of pilot trials in clinical research in critical care. Crit Care Med 2009;37(1 Suppl):S69-74.

21. Chan A-W, Tetzlaff JM, Gotzsche PC, et al. SPIRIT 2013 explanation and elaboration: guidance for protocols of clinical trials. BMJ 2013;346:e7586.

22. Hoffmann TC, Glasziou PP, Boutron I, et al. Better reporting of interventions: template for intervention description and replication (TIDieR) checklist and guide. BMJ 2014;348:g1687.

23. De Jonghe B, Sharshar T, Lefaucheur JP, et al. Paresis acquired in the intensive care unit: a prospective multicenter study. JAMA 2002;288:2859-67.

24. Muscedere J, Dodek P, Keenan S, et al. Comprehensive evidence-based clinical practice guidelines for ventilator-associated pneumonia: prevention. J Crit Care 2008;23:126-37.

25. Sinuff T, Muscedere J, Cook D, et al. Ventilator-associated pneumonia: Improving outcomes through guideline implementation. J Crit Care 2008;23:118-25.

26. Koo K. Survey of acute rehabilitation in Canadian intensive care units. McMaster University, Hamilton, ON, Canada, 2012.

27. Denehy L, Skinner EH, Edbrooke L, et al. Exercise rehabilitation for patients with critical illness: a randomized controlled trial with 12 months of follow-up. Crit Care 2013;17:R156.

28. Berney SC, Harrold M, Webb SA, et al. Intensive care unit mobility practices in Australia and New Zealand: a point prevalence study. Crit Care Resusc 2013;15:260-5.

29. Nydahl P, Ruhl AP, Bartoszek G, et al. Early mobilization of mechanically ventilated patients: a 1-day point-prevalence study in Germany. Crit Care Med 2014;42:1178-86.

30. Hodgson C, Bellomo R, Berney S, et al. The TEAM Study Investigators. Early mobilization and recovery in mechanically ventilated patients in the ICU: a bi-national, multi-centre, prospective cohort study. Crit Care 2015;19:81.

31. Denehy L, de Morton NA, Skinner EH, et al. A physical function test for use in the intensive care unit: validity, responsiveness, and predictive utility of the physical function ICU test (scored). Phys Ther 2013:93:1636-45

32. Skinner EH, Berney S, Warrillow S, et al. Development of a physical function outcome measure (PFIT) and a pilot exercise training protocol for use in intensive care. Crit Care Resusc 2009;11:110-15.

33. Parry SM, Denehy L, Beach LJ, et al. Functional outcomes in ICUwhat should we be using? - an observational study. Crit Care 2015:19:127.

34. Fan E, Ciesla ND, Truong AD, et al. Inter-rater reliability of manual muscle strength testing in ICU survivors and simulated patients. Intensive Care Med 2010;36:1038-43.

35. Hermans G, Clerckx B, Vanhullebusch T, et al. Interobserver agreement of Medical Research Council sum-score and handgrip strength in the intensive care unit. Muscle Nerve 2012;45:18-25.

36. Vanpee G, Segers J, Van Mechelen $\mathrm{H}$, et al. The interobserver agreement of handheld dynamometry for muscle strength assessment in critically ill patients. Crit Care Med 2011;39:1929-34.

37. Jones CJ, Rikli RE, Beam WC. A 30-s chair-stand test as a measure of lower body strength in community-residing older adults. Res $Q$ Exerc Sport 1999;70:113-19.

38. Tveter AT, Dagfinrud H, Moseng T, et al. Health-related physical fitness measures: reference values and reference equations for use in clinical practice. Arch Phys Med Rehabil 2014;95:1366-73.

39. Bohannon RW, Wang Y-C, Gershon RC. Two-minute walk test performance by adults 18 to 85 years: normative values, reliability, and responsiveness. Arch Phys Med Rehabil 2015;96:472-7.

40. Pin TW. Psychometric properties of 2-minute walk test: a systematic review. Arch Phys Med Rehabil 2014;95:1759-75.

41. Kho ME, Truong AD, Brower RG, et al. Neuromuscular electrical stimulation for intensive care unit-acquired weakness: protocol and methodological implications for a randomized, sham-controlled, phase II trial. Phys Ther 2012;92:1564-79.

42. Parry SM, Berney S, Koopman R, et al. Early rehabilitation in critical care (eRiCC): functional electrical stimulation with cycling protocol for a randomised controlled trial. BMJ Open 2012:2:e001891.

43. Bagshaw SM, Stelfox HT, McDermid RC, et al. Association between frailty and short- and long-term outcomes among critically ill patients: a multicentre prospective cohort study. CMAJ 2014;186:E95-102.
44. Katz S, Ford AB, Moskowitz RW, et al. Studies of illness in the aged. The index of Adl: a standardized measure of biological and psychosocial function. JAMA 1963;185:914-19.

45. Wade DM, Hankins M, Smyth DA, et al. Detecting acute distress and risk of future psychological morbidity in critically ill patients: validation of the Intensive care psychological assessment tool. Crit Care 2014:18:519.

46. Wade DM, Howell DC, Weinman JA, et al. Investigating risk factors for psychological morbidity three months after intensive care: a prospective cohort study. Crit Care 2012;16:R192.

47. Herdman M, Gudex C, Lloyd A, et al. Development and preliminary testing of the new five-level version of EQ-5D (EQ-5D-5L). Qual Life Res 2011;20:1727-36.

48. van Hout $B$, Janssen MF, Feng Y-S, et al. Interim scoring for the EQ-5D-5L: mapping the EQ-5D-5L to EQ-5D-3L value sets. Value Health 2012;15:708-15.

49. Xie F, Pullenayegum E, Gaebel K, et al. A time trade-off-derived value set of the EQ-5D-5L for Canada. Med Care 2016;54:98-105.

50. Sricharoenchai T, Parker AM, Zanni JM, et al. Safety of physical therapy interventions in critically ill patients: a single-center prospective evaluation of 1110 intensive care unit admissions. J Crit Care 2014;29:395-400.

51. Kho ME, Martin RA, Toonstra AL, et al. Le Tour De ICU: feasibility and safety of routine use of in-bed cycling for physical rehabilitation in the intensive care unit. Am J Respir Crit Care Med 2013;187: A3880.

52. Pires-Neto RC, Pereira AL, Parente $\mathrm{C}$, et al. Characterization of the use of a cycle ergometer to assist in the physical therapy treatment of critically ill patients. Rev Bras Ter Intensiva 2013;25:39-43.

53. Hickmann CE, Roeseler J, Castanares-Zapatero D, et al. Energy expenditure in the critically ill performing early physical therapy. Intensive Care Med 2014;40:548-55.

54. Dantas CM, Silva PF, Siqueira FH, et al. Influence of early mobilization on respiratory and peripheral muscle strength in critically ill patients. Rev Bras Ter Intensiva 2012;24:173-8.

55. Knaus WA, Draper EA, Wagner DP, et al. APACHE II: a severity of disease classification system. Crit Care Med 1985;13:818-29.

56. Charlson ME, Pompei P, Ales KL, et al. A new method of classifying prognostic comorbidity in longitudinal studies: development and validation. J Chronic Dis 1987:40:373-83.

57. Groll DL, To T, Bombardier C, et al. The development of a comorbidity index with physical function as the outcome. J Clin Epidemiol 2005;58:595-602.

58. Needham DM, Wozniak AW, Hough CL, et al. Risk factors for physical impairment after acute lung injury in a national, multicenter study. Am J Respir Crit Care Med 2014;189:1214-24.

59. de Jonghe B, Lacherade J-C, Sharshar T, et al. Intensive care unit-acquired weakness: risk factors and prevention. Crit Care Med 2009;37(10 Suppl):S309-15

60. Cocks K, Torgerson DJ. Sample size calculations for pilo randomized trials: a confidence interval approach. $J$ Clin Epidemiol 2013;66:197-201.

61. Cooke CR, Erickson SE, Watkins TR, et al. Age-, sex-, and race-based differences among patients enrolled versus not enrolled in acute lung injury clinical trials. Crit Care Med 2010;38:1450-7.

62. Casey $\mathrm{CM}$. The study of activity in older ICU patients: an integrative review. J Gerontol Nurs 2013;39:12-25; quiz 26-7.

63. Editors. ICoMJ. Recommendations for the Conduct, Reporting, Editing, and Publication of Scholarly work in Medical Journals. Secondary Recommendations for the Conduct, Reporting, Editing, and Publication of Scholarly work in Medical Journals. 2010. http:// www.icmje.org/recommendations/.

64. LeBlanc AD, Schneider VS, Evans HJ, et al. Regional changes in muscle mass following 17 weeks of bed rest. J Appl Physiol 1992;73:2172-8.

65. Gallagher D, Visser M, De Meersman RE, et al. Appendicular skeletal muscle mass: effects of age, gender, and ethnicity. J Appl Physiol 1997;83:229-39.

66. Ferrando AA, Stuart CA, Brunder DG, et al. Magnetic resonance imaging quantitation of changes in muscle volume during 7 days of strict bed rest. Aviat Space Environ Med 1995;66:976-81.

67. Needham DM, Truong AD, Fan E. Technology to enhance physical rehabilitation of critically ill patients. Crit Care Med 2009;37(10 Suppl):S436-41.

68. Kho ME, Molloy AJ, McCaughan M, et al. TryCYCLE: preliminary results of early in-bed cycling with mechanically ventilated patients. $J$ Crit Care 2015;30:1419.

69. Needham DM, Bronskill SE, Calinawan JR, et al. Projected incidence of mechanical ventilation in Ontario to 2026: preparing for the aging baby boomers. Crit Care Med 2005;33:574-9. 